\title{
Low serum 25-Hydroxy $(\mathrm{OH})$ vitamin D levels are associated with increased arterial stiffness in healthy children: An echocardiographic study from Turkey
}

\author{
Emine Azak $^{1}$ and İlker Çetin ${ }^{2}$ \\ ${ }^{1}$ University of Health Sciences, Ankara City Hospital \\ ${ }^{2}$ Yildirim Beyazit University Faculty of Medicine
}

May 16, 2021

\begin{abstract}
Objective: Arterial stiffness refers to arterial wall rigidity, particularly in central vessels, and is an independent predictor of cardiovascular disease. 25-Hydroxy $(\mathrm{OH})$ vitamin D has beneficial effects on blood pressure, vascular endothelial function, and arterial stiffness. Therefore, we aimed to elucidate the role of $25-\mathrm{OH}$ vitamin D deficiency in arterial stiffness development and its relationship with arterial stiffness in healthy children. Methods: This study included 80 patients with low levels of 25 -OH vitamin D and 40 healthy control. The study participants were then divided into three groups: group 1 consisted of patients with a deficient 25-OH D level of $<19.9 \mathrm{ng} / \mathrm{mL}$, group 2 with an insufficient 25-OH D level between 20-29.9 ng/mL; group 3 were considered control group with a sufficient serum $25-\mathrm{OH}$ vitamin D level of [?]30 ng/mL. Aortic strain, distensibility, stiffness index, and standard left ventricular measurements were calculated using M-mode echocardiographic data. Results: Left ventricular mass index (LVMI) and interventricular septal diastolic thickness (IVSTd) appeared to increase in group 1 compared to groups 2 and 3. Aortic strain and distensibility were significantly decreased in group 1, whereas aortic stiffness index and elastic modulus were significantly increased. The aortic stiffness index was negatively correlated with serum $25-\mathrm{OH}$ vitamin D levels; however, aortic strain, aortic distensibility, and LVMI were positively correlated. Conclusions: Our study results revealed a significant relationship between $25-\mathrm{OH}$ vitamin D levels indicative of a deficiency and aortic stiffness. We suggest that arterial stiffness may also occur in healthy children with a $25-\mathrm{OH}$ vitamin D deficiency.
\end{abstract}
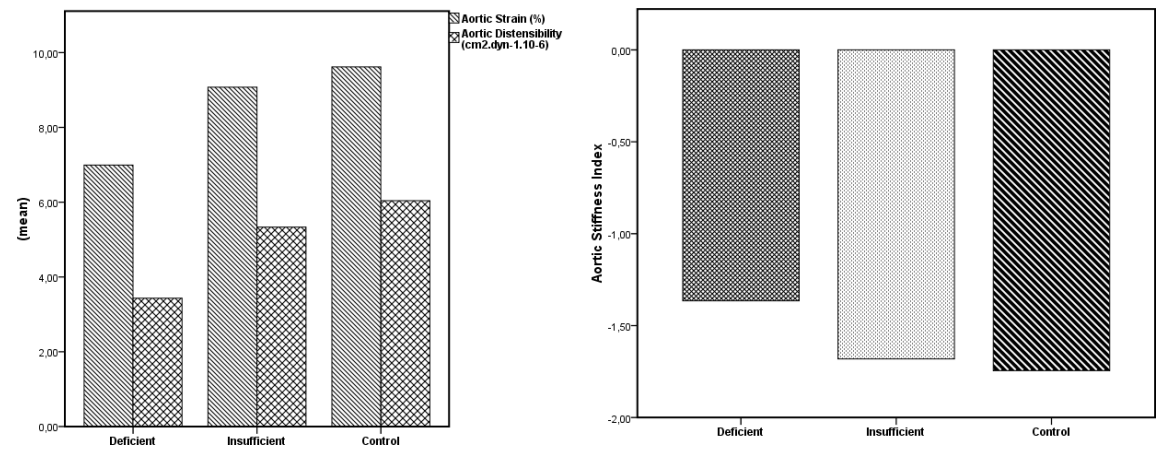

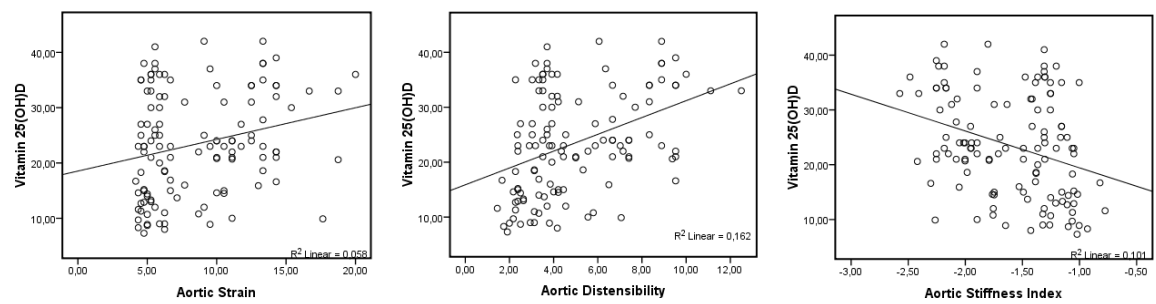